\title{
Chemo-population multizone models for emission line evolution of star forming galaxies
}

\author{
G. Valle ${ }^{1}$ and S. N. Shore ${ }^{1,2}$ \\ 1 Dipartimento di Fisica "Enrico Fermi”, Università di Pisa, largo Pontecorvo 3, Pisa 56127, Italy \\ e-mail: [valle; shore]@df.unipi.it \\ 2 INFN/Pisa, largo Pontecorvo 3, Pisa 56127, Italy
}

Received 24 April 2006 / Accepted 31 October 2006

ABSTRACT

\begin{abstract}
Aims. We apply the nonlinear chemo-population (CP) models for galactic star formation and abundance evolution to the prediction of emission line diagnostics.

Methods. We interfaced the CP code with the photoionization code Cloudy. Using the self-consistently generated star forming rates and abundance histories we predict the time dependences of the nebular line emissivities from model H II regions. We also simulated some effects of collisions, stripping, and accretion events on the emission line diagnostics.

Results. We find, despite using extremely simplified input fluxes (i.e. blackbodies scaled to a range of effective temperatures but without detailed model atmospheres or population synthesis), the models reproduce many features of the observations. In particular, we suggest that the line ratios for highly ionized species (e.g. [O III] vs. [N II]) are more sensitive to the galactic history, while the exclusively ionization diagnostics (e.g. [O II] vs. [O I]) are more sensitive to the underlying stellar population and the composite ultraviolet flux distribution. We discuss some reasons for this and conclude that a coupled treatment of the galactic population and chemical evolution is essential for interpretation of the data. We also find that collisional and stripped models cover a wider range in the diagnostic diagrams that provide clues to the environmental effects.
\end{abstract}

Key words. galaxies: evolution - galaxies: abundances - galaxies: interactions

\section{Introduction}

One of the basic cosmological questions is the history of star formation over cosmic time. It is studied by observing the luminosity of $\mathrm{H}$ II region emission lines produced by massive hot stars within galaxies. These short-lived OB stars, inside or in the vicinity of gas-rich regions, emit ultraviolet photons that ionize the gas. The resulting recombination emission lines provide a proxy measurement of the star formation rate (SFR) in the integrated galactic spectrum. The strongest and most frequently used lines are those of the Balmer series, particularly $\mathrm{H} \alpha 6563 \AA$. Their intensity is, to a first approximation, independent of the metallicity of the gas and unlike the Lyman series, they are optically thin. Since the line emission strength depends only on the rate of recombination (hence the ambient density) and the ionization rate (hence, the stellar luminosity and ambient density) it is possible to convert the $\mathrm{H} \alpha$, and other nebular line strengths (e.g. other commonly observed optical lines are [N II] $\lambda 6548$, 6584, [O II] $\lambda$ 3727, [O III] $\lambda$ 4959, 5007, He II $\lambda$ 4686, etc.) into SFRs using semi-empirical calibrations (cf. Kennicutt 1983, 1998). To extract information on the star forming activity from these indicators, however, it is also necessary to know something about the chemical history of the gas. In the previous papers in this series (Valle et al. 2002; Valle et al. 2005a, hereafter VSG05; Valle et al. 2005b, hereafter VCS05) we presented results for light element synthesis, interacting systems, and finally the interpretation of synthetic color-magnitude diagrams (CMD) resulting from the population evolution within the model systems. In this last paper in the series, we show how to model the time development of the emission lines by combining the galactic chemo-population code with the widely used photoionization code Cloudy (Ferland 1996).

In Sect. 2 we describe our methods, in Sect. 3 we present some of the results compared with observations under very simple conditions, and discuss some interpretive implications in the final section.

\section{Merging the Galactic evolution code with Cloudy}

We used the multi-zone, multi-population model of Galactic evolution (chemo-population models, hereafter $\mathrm{CP}$, to distinguish them from the complementary, but different, chemodynamical approach, see Rocha-Pinto et al. 2004) that has been described in detail in earlier papers (Shore \& Ferrini 1995, Valle et al. 2002) to compute star formation histories and time dependent heavy element yields. These furnished the metal abundances for the diffuse and molecular gas phases to serve as input to the photoionization code Cloudy, with which we computed the emission line strengths and ionization structures for the thin and thick disk diffuse gas. Some effects of collisions, stripping, and accretion events on the emission line diagnostics were also modeled to complete the connection with the previous studies.

The CP approach represents in approximate fashion the various galactic components (e.g. diffuse gas, molecular clouds, stars, etc.) as populations that can interact and inter-convert. The rates for the various processes are the same we have used in our previous papers. This dynamical systems approach simultaneously solves the time development of the individual "phases" of a model galaxy (gas, $g$, clouds, $c$, and stars, $s$, in different mass ranges) within a system of equations that allow complete 
inter-phase coupling. We obtain the time dependent star formation rate, $\psi$, from the $\mathrm{CP}$ code assuming neither instantaneous recycling nor any particular fixed infall or outflow law for the diffuse gas. The galactic structure is approximated using three zones that are able to exchange mass: halo (HA), thick disk (TD), thin disk (DI). In VSG05 we extended the code to include stochastic effects simulating stripping, accretion from outside the system, and collisional suppression of star formation.

In a one zone treatment, the gas fraction and time are interchangeable even if there is infall, provided the rate of mass accretion is a monotonic decreasing function of time (e.g. Köppen $\&$ Hensler 2005). Although we show the results here only for the disk, the $\mathrm{CP}$ code treats the galactic evolution with all the three zones exchanging mass and heavy elements. Thus, the gas fraction does not strictly decrease with time because the disk is a secondary structure and at later epochs is self-consistently replenished by mass lost from the halo to the combined thin and thick disks. This breaks the conjugate relation between the gas fraction and time. We made, however, a significant change in the usual prescription of the gas compared to our earlier work. In the standard model and its extension (VSG05) the results have been given for each of the model zones without complete mixing among them. The contribution of the diffuse gas belonging to the thin and thick disks were treated here with the algorithm:

$g(t)=g_{\mathrm{DI}}(t)+g_{\mathrm{TD}}(t)\left(h_{\mathrm{DI}} / h_{\mathrm{TD}}\right)$

where $g_{\mathrm{DI}}$ and $g_{\mathrm{TD}}$, are, respectively, the gas phases of the thin and thick disks and $h_{\mathrm{DI}}$ and $h_{\mathrm{TD}}$ are their respective scale heights. In the simulations we adopted $h_{\mathrm{DI}}=100 \mathrm{pc}$ and $h_{\mathrm{TD}}=300 \mathrm{pc}$. The metallicity for the gas was computed weighting the thin $\left(Z_{\mathrm{DI}}\right)$ and the thick $\left(Z_{\mathrm{TD}}\right)$ disk contribution according to the gas phase:

$Z_{h_{\mathrm{DI}}}(t)=\left[Z_{\mathrm{DI}}(t) g_{\mathrm{DI}}(t)+Z_{\mathrm{TD}}(t) g_{\mathrm{TD}}(t)\left(h_{\mathrm{DI}} / h_{\mathrm{TD}}\right)\right] / g(t)$.

Figure 1 shows the evolution of the combined gas phase and of the combined metallicity, obtained from Eq. (1) and Eq. (2), both with the gas and metallicity of the thin and the thick disk zones. The abundances histories of the elements were inserted into Cloudy using Eq. (2). The gas number density, $n(z, t)$, follows from:

$n(z, t)=n_{0}\left[\operatorname{sech}^{2}\left(z / h_{\mathrm{DI}}\right)+\operatorname{sech}^{2}\left(z / h_{\mathrm{TD}}\right)\right] \frac{g(t)}{g\left(t_{\max }\right)}$

where $t_{\max }$ is the time for which the quantity $g(t)$ in Eq. (1) assumes its maximum value. The value $n_{0}\left(\right.$ in $\left.\mathrm{cm}^{-3}\right)$, is obtained from the normalization:

$n_{0}=\frac{n_{\mathrm{m}} h_{\mathrm{DI}}}{\int_{0}^{h_{\mathrm{DI}}}\left[\operatorname{sech}^{2}\left(z / h_{\mathrm{DI}}\right)+\operatorname{sech}^{2}\left(z / h_{\mathrm{TD}}\right)\right] \mathrm{d} z} ;$

since it is a free parameter when normalized, we chose $n_{\mathrm{m}}=$ $100 \mathrm{~cm}^{-3}$ as a typical density value for an H II region for our standard case but for comparison, in the following section, we show the results for lower and higher densities, setting $n_{\mathrm{m}}=5$, $10,10^{3}$, and $10^{4} \mathrm{~cm}^{-3}$.

To represent the luminosity history for the ionizing stars in Cloudy, we followed the usual procedure to scale the output SFR for a fixed initial mass function (IMF) to the number of high mass stars at any moment. We then scaled the Lyman continuum luminosity of the emitting source with the SFR of the thin disk: $L\left(v \geq v_{L y C}, t\right) \propto \psi_{\mathrm{DI}}(t)$. In the calculations, all halo star formation ceased after about $1 \mathrm{Gyr}$ and was also significantly lower in the thick disk. Only the thin disk remains active. The peak of
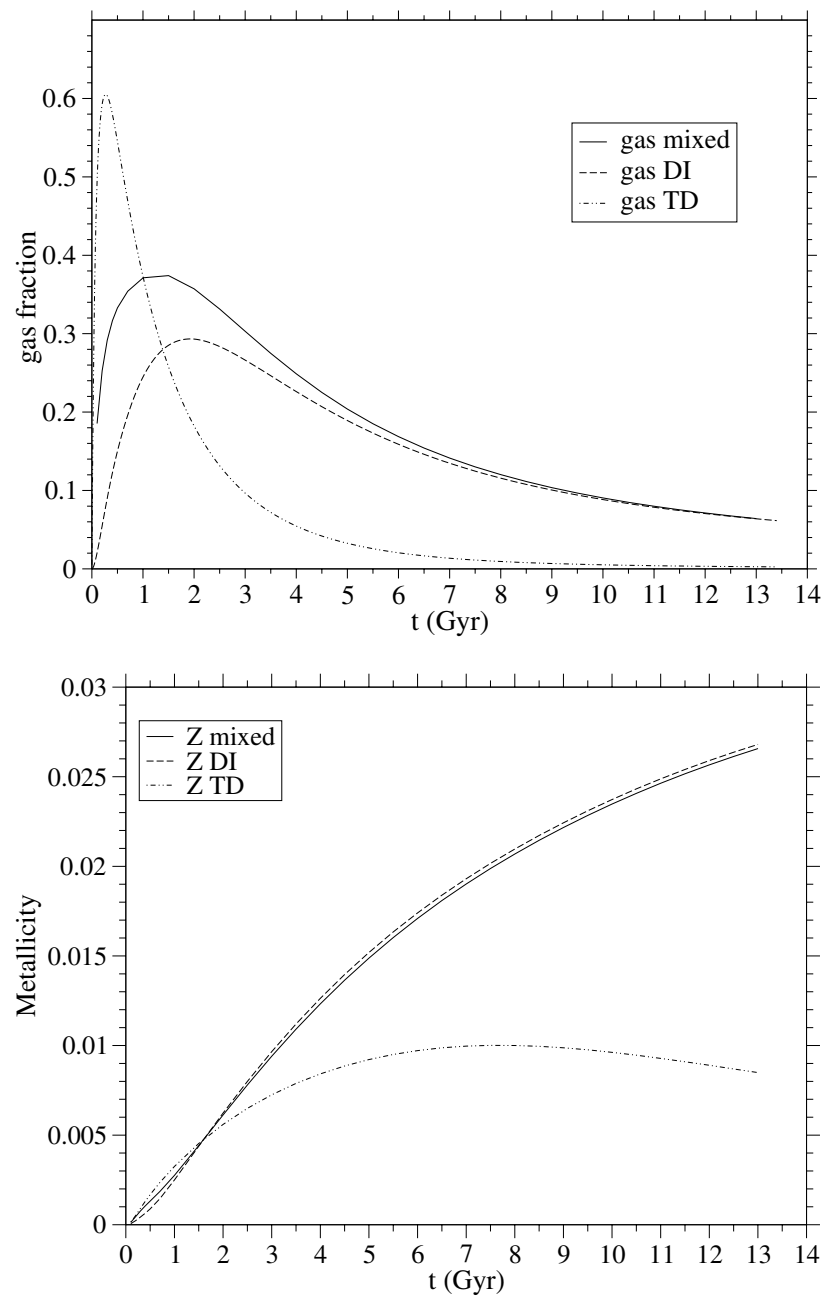

Fig. 1. Gas fraction vs. time a) and metallicity vs. time b) in the standard case of our galactic evolution code, with the mixing of the results for the thin and the thick disk zones, following Eqs. (1) and (2).

the luminosity at the maximum SFR was set equal to the luminosity of an $\mathrm{OB}$ association of $\approx 100$ stars but this number is an arbitrary, and not a critical, choice. For the next section, we refer to the following choices for the stellar properties as separate cases $^{1}$ :

- Case (i): O6 III stars with $T_{1, \text { eff }}=50960 \mathrm{~K}$;

- Case (ii): O3 III stars with $T_{2 \text {,eff }}=42640 \mathrm{~K}$

- Case (iii): 09.5 V stars with $T_{3, \text { eff }}=34620 \mathrm{~K}$.

In all cases we approximated the input radiative source as a single blackbody at fixed temperature. The stellar population is fixed, i.e. we use the same spectral type for the stars, varying in number formed during the history according to the IMF and the SFR obtained from the CP code (see Fig. 2a). We are consequently neglecting the changes in opacity occurring during the evolution of the chemical composition of the galaxy and the opacity effects that modify the UV spectrum of the OB stars. As Balick and Sneden (1976) pointed out, the increase of the metallicity implies a decrease of the relative abundances of the high ionization stages in the $\mathrm{H}$ II region; consequently the determinations of metal abundances in the nebula can be affected. In a similar vein, another simplifying assumption was to neglect dust (see below).

1 These uses the calibrations cited in Shore (2003, Table 6.11) and Vacca et al. (1996). 

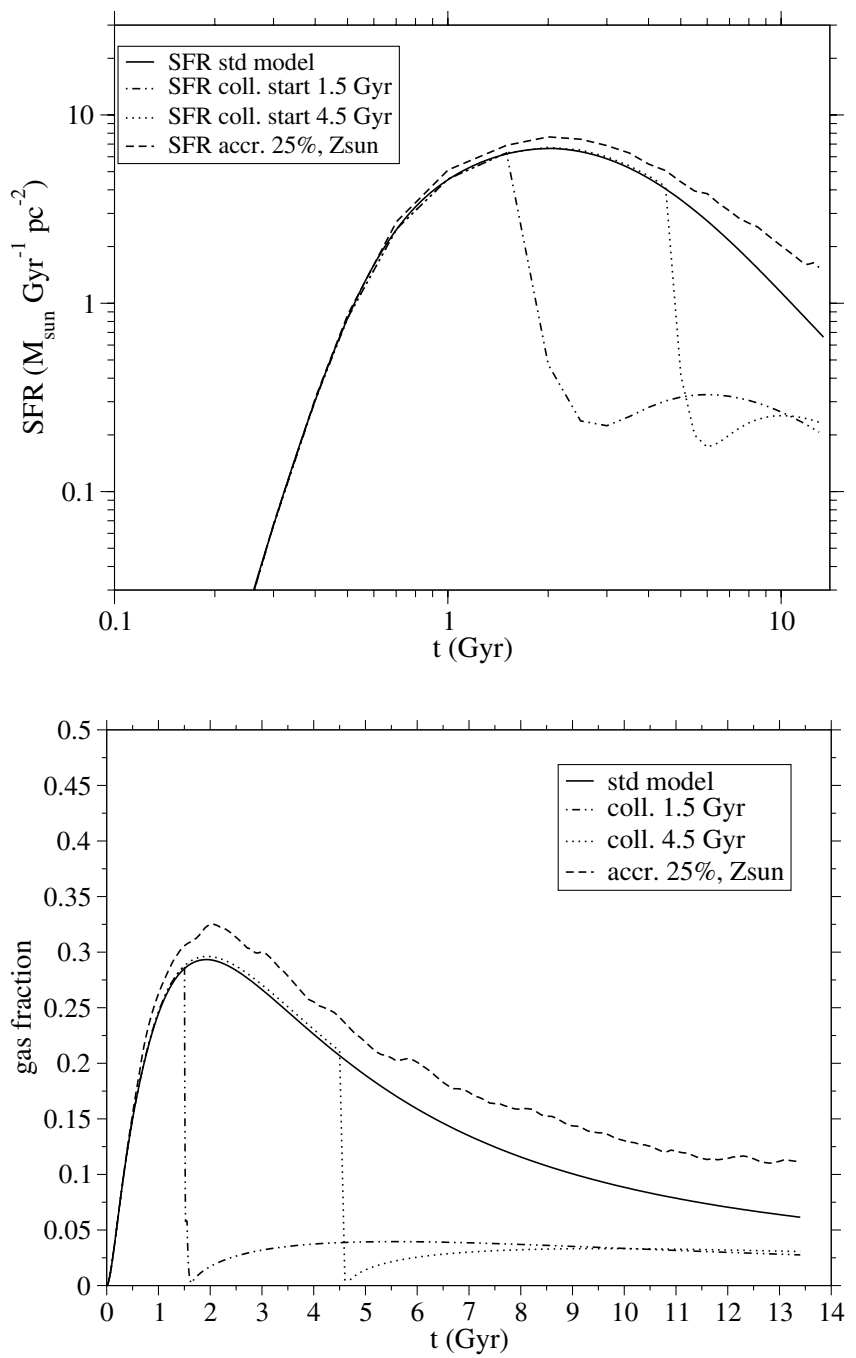

Fig. 2. SFR for the thin disk (a), top) and gas fraction $g_{\mathrm{DI}}(\mathbf{b})$, bottom) from the CP code in the cases: (black continuous) standard model; (dotdash) collision, with $t_{\text {start }}=1.5 \mathrm{Gyr}$ and $\Delta t_{\text {coll }}=130 \mathrm{Myr}$; (dots) collision, with $t_{\text {start }}=4.5 \mathrm{Gyr}$ and $\Delta t_{\text {coll }}=130 \mathrm{Myr}$; (dash) accretion with metal rich ambient gas of the $25 \%$ of the initial mass.

We used a spherical geometry with a filling factor equal to unity (homogeneous gas layers). We chose $1 \mathrm{pc}$ as the inner radius of the sphere but in the final diagnostic plots we also include a version of case (i) with an inner radius of $100 \mathrm{pc}$ to model a more dilute hot interstellar diffuse radiation field that may be expected in, for instance, a starburst galaxy. In our simulation the radius of the sphere corresponds to the scale height of the disk so its range is quite narrow, depending on the assumption on $h_{\mathrm{DI}}$. The density profiles in Cloudy can only be implemented as a variation in the depth in the cloud or the distance from the source (so $r$ ) and not the height $(z)$. In other words, no two dimensional density profiles are included, Cloudy approximates cylindrical geometry as a truncated sphere.

A number of recent investigations have studied emission line predictions from star forming galaxies, frequently concentrating on H II galaxies, starbursts, and irregulars. The population synthesis code PEGASE2 (Fioc \& Rocca-Volmerange 1999) has been mated to Cloudy (Moy, Rocca-Volmerange, \& Fioc 2001) to compute emission line diagnostics. In this approach, a variety of functional forms for the time dependent SFR and IMF can be selected along with a range of metallicities, although in published studies an age-metallicity relation is not used. The STARBURST99 code (e.g. Kewley et al. 2001, 2004) works similarly but has been combined with a different nebular code, MAPPINGS3 (e.g. Sutherland \& Dopita 1993) using specific functional representations for the histories of the relevant galactic properties and then forming detailed composite spectral energy distributions from libraries (e.g. Leitherer et al. 1999; Martins et al. 2005). Stasinska \& Izotov (2003) couple a different photoionization code with various stellar continuum models depending on the IMF and star forming history. Zackrisson et al. (2001) do not explicitly treat the chemical evolution and use a single stellar population evolving at constant metallicity formed according to an adopted initial mass function that follows a power law in the selected mass range.

These previous calculations differ from ours in two important senses: the SFR is assumed a priori and the metallicity is generally kept constant. For instance, Moy et al. (2001) chose a variety of laws, Maier et al. (2006) use exponentials or combinations of exponentials with power laws and also include infall for dilution. Recently, however, van Zee \& Haynes (2006) compared the predictions of closed box models along with accretion or wind outflow modifications with $\mathrm{O}$ and $\mathrm{N}$ emission line diagnostics for irregular galaxies and Maier et al. (2006) used similar models for the evolution of the oxygen abundances at high redshift. A variety of other physical properties differ between the models, e.g. the treatment of dust, filling factors for the diffuse gas, and geometries, but the basic assumptions are similar for the galactic evolution, the star formation and metallicity histories are usually treated separately; the exception is the closed box model which makes unique predictions for the correspondence of yield with diffuse gas mass fraction. In contrast, we have treated in more detail the galactic evolution and greatly simplified the radiative inputs. Before presenting our results, we wish to make a basic point about our modeling procedure. The agreement we will show is actually better than might be expected considering the simplifications we made for the photoionization modeling. We show, however, that several standard diagnostics are actually quite robust and relatively insensitive to the details of the models.

\section{Simulations}

\section{1. $L(H \alpha)$ time evolution and linearity with SFR}

The $\mathrm{H} \alpha$ luminosity dependence on the star formation rate is the best known, and most reliable, empirical relation (Kennicutt 1983). It seems to apply to all types of galaxies that are actively forming stars and is a simple linear relationship (e.g. Kennicutt 1998). Obviously our standard model must reproduce this, but we have also explored the consequences of the other scenarios we have treated in this series to see if they produce departures from the linear relationship. We chose some representative cases from those studied in VSG05: (a) the standard model; (b) a single collision, lasting of $130 \mathrm{Myr}$ starting arbitrarily at $1.5 \mathrm{Gyr}$, using a mass loss history within the stripping during the collision obtained numerically by Quilis et al. (2000); (c) a single collision, with the same characteristics as (b) but starting arbitrarily at $4.5 \mathrm{Gyr}$; and (d) stochastic accretion of $25 \%$ of the initial mass due to environmental infall of material with solar composition. The SFR and gas fraction content of these four situations are shown in Fig. 2, in which only the thin disk evolution is displayed. Despite the complexity of line formation from diffuse gas within an actively star-forming system, there is evidence that $\mathrm{H} \alpha$ and the optical line ratios have values similar to those in 

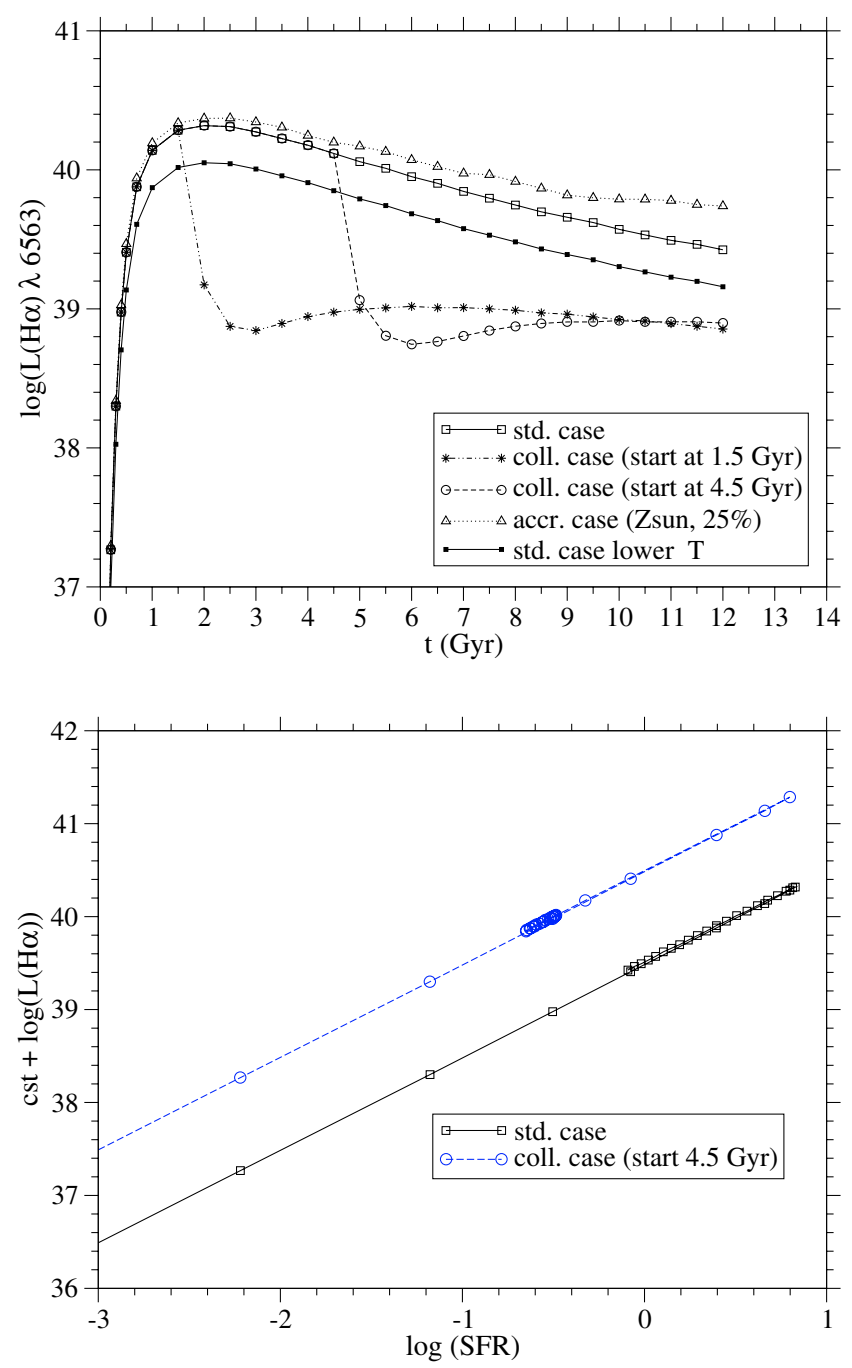

Fig. 3. a) $\log (\mathrm{L}(\mathrm{H} \alpha))$ vs. time in five cases: (black continuous, squares) standard model; (dot-dash, stars) collision, with $t_{\text {start }}=1.5 \mathrm{Gyr}$ and $\Delta t_{\text {coll }}=130 \mathrm{Myr}$; (dash, circles) collision, with $t_{\text {start }}=4.5 \mathrm{Gyr}$ and $\Delta t_{\text {coll }}=130 \mathrm{Myr}$; (dot, triangles) metal-rich $\left(Z=Z_{\odot}\right)$ stochastic accretion by 25\%; (black continuous, filled squares) standard model, with lower temperature $(T=42640 \mathrm{~K})$. b) $\log (\mathrm{L}(\mathrm{H} \alpha))$ vs. $\log (\mathrm{SFR})$ : (black continuous, squares) standard model; (dashed, blue circles) collision starting at 4.5 Gyr. For the displayed models, the corresponding SFR vs. time is shown in Fig. 2a and their $\log (\mathrm{L}(\mathrm{H} \alpha))$ vs. time is in a). The conditions of the simulations are explained in Sect. 2, case (i). To show the relative population of the different regions for both cases, the two lines have been displaced by a constant, otherwise they would coincide. The most populated region in the collision model does not correspond to the densest ensemble of points from the standard model at the same times of the galactic evolution.

the spectra of individual H II regions (see Lehnert \& Heckman 1994; Kennicutt 1998; Kobulnicky et al. 1999).

Figure 3 a shows the time development of the $\mathrm{H} \alpha$ luminosity. The maximum corresponds to the peak SFR for the disk, at about 2-3 Gyr (see Fig. 2). Figure 3b shows the $\mathrm{H} \alpha$ luminosity vs. the SFR. It displays the standard model and the collision starting at $4.5 \mathrm{Gyr}$, in case (i), reproducing the well established linear relation in both cases, also for the accretion and the early epoch collision models. Since the two cases lie on the same line, we have displaced them by an arbitrary constant. Note that we do not determine the proportionality constant, the precise value of which depends on the Ly continuum flux and the IMF, among other
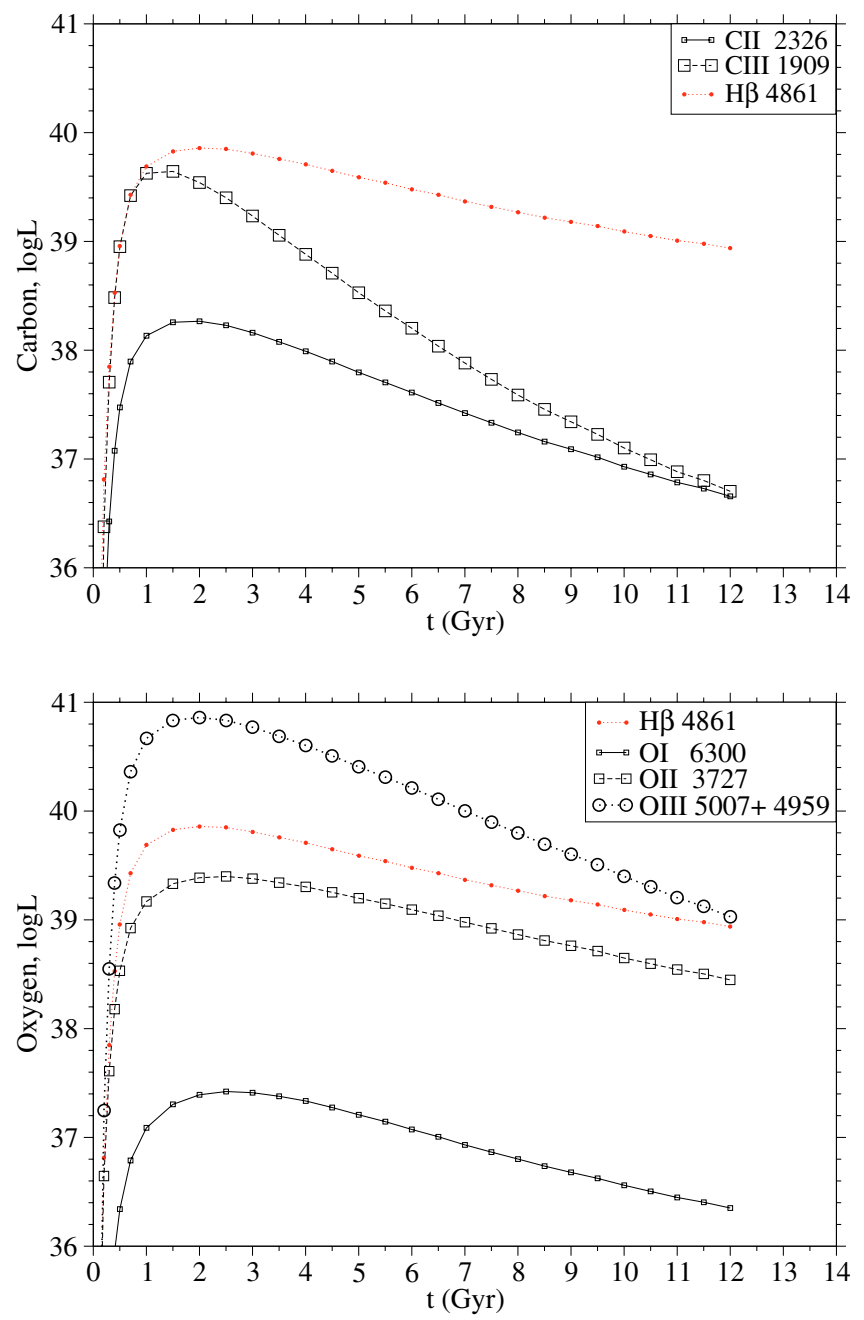

Fig. 4. Luminosities (erg/s) vs. time (Gyr) of carbon (a), top) and oxygen (b), bottom), in the standard model, case (i). For comparison $\mathrm{H} \beta(\lambda 4861)$ evolution is also displayed.

factors. With a time IMF, the star formation always yields the same fraction of stars of a specific effective temperature while the spectral energy distribution continues to redden as the lower mass stars become more numerous and the higher masses evolve. This changes the stellar colors but not the emission line diagnostics. Since the proportionality coefficient depends on the efficiency with which far-UV radiation is converted to $\mathrm{H} \alpha$ emission, it should depend on the geometry of the gas layer the presence of dust, etc. But in all cases, the $\mathrm{H} \alpha$ emission is a photon counter and is therefore ultimately linear in the SFR.

To emphasize this, we note that regardless of the model scenario - standard models, collisions, or stripping - the same linear law holds. The principal difference is the range exhibited in $\mathrm{H} \alpha$ luminosity and SFR for the different cases. As expected, the collisional models lie along the same line but occupy only a very small range because star formation is suppressed for the bulk of their history. The metallicity evolution, which can differ dramatically among the models, produces almost no effect on the formal law.

\subsection{Other emission line diagnostics}

Other emission line ratios have been used as diagnostics of star formation (e.g. Moustakas et al. 2005, Kennicutt 1998). In the 

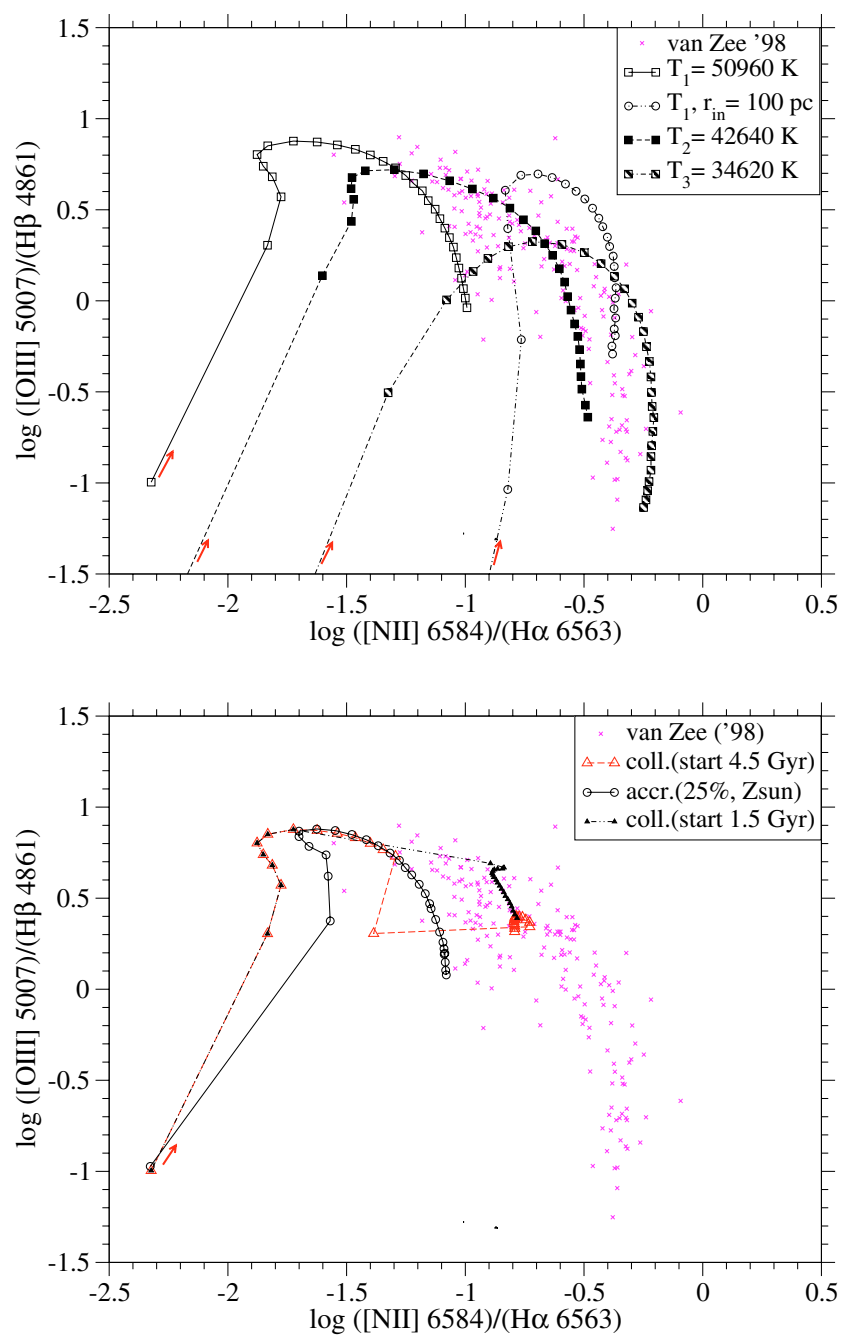

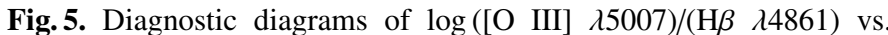
$\log ([\mathrm{N} \mathrm{II}] \lambda 6584) /(\mathrm{H} \alpha$ 16563), compared with the observations of H II region in spiral galaxies, from van Zee et al. (1998) (magenta crosses). Each model is a sequence in time, the red arrow indicates the sequence from early to later times of the simulation. The highest values of $\log [\mathrm{O} \mathrm{III]} /(\mathrm{H} \beta)$ correspond to the peak of the SFR for the standard model, as in Fig. 2. Symbols: (black continuous, squares) standard model, case (i) of Sect. 2; (dot-dash, circles) standard model, case (i) of Sect. 2, but with inner radius in the Cloudy simulation of $100 \mathrm{pc}$; (dash, filled squares) standard model, case (ii) of Sect. 2; (dot-dash, semifilled squares) standard model, case (iii) of Sect. 2; (dot-dash, black filled triangles) collision, with $t_{\text {start }}=1.5 \mathrm{Gyr}$ and $\Delta t_{\text {coll }}=130 \mathrm{Myr}$, case (i) of Sect. 2; (dash, red triangles) collision, with $t_{\text {start }}=4.5 \mathrm{Gyr}$ and $\Delta t_{\text {coll }}=130 \mathrm{Myr}$, case (i); (black continuous, circles) metal-rich $\left(Z=Z_{\odot}\right)$ stochastic accretion by $25 \%$, case (i). Figures in color available in the electronic version of the paper.

following, Figs. 4a,b show the evolution with time of the luminosity of carbon and oxygen emission lines in case (i) for the standard model; Figs. 5a,b give the diagnostic diagrams of $[\mathrm{O}$ III] $(\lambda$ 5007)/H $\beta(\lambda$ 4861) vs. [N II] $(\lambda$ 6584)/H $\alpha(\lambda$ 6563), and Fig. 6 shows (a) $[\mathrm{O} \mathrm{III}] / \mathrm{H} \beta$ vs. $[\mathrm{O} \mathrm{II}](\lambda \quad 3727) /[\mathrm{O}$ III $]$, (b) $[\mathrm{O}$ III $] / \mathrm{H} \beta$ vs. $[\mathrm{O} \mathrm{II}] / \mathrm{H} \beta$, and (c) $[\mathrm{O} \mathrm{III}] / \mathrm{H} \beta(\lambda$ 4861) vs. [O I] $(\lambda$ 6300)/H $\alpha$. In these figures the standard, accretion, and collisions models - coupled to Cloudy with the density, temperature and luminosity conditions in case (i) $\left(T_{1}=50960 \mathrm{~K}\right)$ of Sect. 2 - are displayed; the standard model is also computed in cases (ii) and (iii), i.e. for lower stellar effective temperature ( $T_{2}=42640 \mathrm{~K}$ and $T_{3}=34620 \mathrm{~K}$ ) of the emitting source and in case (i) but for larger inner radius of the nebula $\left(r_{\text {in }}=100 \mathrm{pc}\right)$. Each symbol in Figs. 5 and 6 refers to a single time step of the models previously calculated (standard, collision, accretion), so the sequences shown in these plots are sequences in time.

We also show a comparison between our models and the data from van Zee et al. (1998) (their Table 5, that lists a sample of $\mathrm{H}$ II regions in different spiral galaxies). In Fig. 5a, superimposing the observations and the models, we note that the sequence in time follows the trend of observations without further scaling despite the neglect of dust in the calculations. The standard model sequence is shown in the figure for the three different source temperatures, and for the standard case with the greater inner radius for the $\mathrm{H}$ II region, an approximation of a more dilute incident radiation field. Our results in Fig. 5a show a good agreement when compared with Fig. 2 of Moustakas et al. (2005). In this recent work, the authors model starburst emission spectra with the PEGASE 2 and STARBURST99 codes, as in Kewley et al. (2001, 2004). Furthermore, the points of our simulations in Figs. 5a,b match the observed galaxies, H II region-like objects, shown in Fig. 14.2 of Osterbrock \& Ferland (2006), and avoid the region containing active galactic nuclei (AGN).

Figure $5 \mathrm{~b}$ shows results for the collision and accretion cases. It is interesting that the particular history experienced by a galaxy could lead to a selection effect on its observability in that the post-collision cases shown cross the data, while the corresponding model at the same times as the standard, or even accretion, models is at the boundary of the observations. The upper part of the diagram, where $\log [\mathrm{O}$ III $] / \mathrm{H} \beta \geq 0.2$ and $\log [\mathrm{N} \mathrm{II}] / \mathrm{H} \alpha \geq-1$, is populated by the post-collision models (black filled triangles and red triangles). This could be a region populated by interacting galaxies, for instance in a cluster, while they avoid the lower part of the diagram, where only the standard models live, and which is likely populated by isolated galaxies. Moreover, comparing Figs. 5a and b, there is an overlap between the post-collisional cases and the standard model case corresponding to the lower source temperature (case (ii)); this overlap resembles the result in our previous paper (VCS05), i.e. if the star formation ceases and then begins again, this can be interpreted as a burst. From the line ratio diagnostics, a burst cannot be uniquely identified. Note that each point of each track in time is a local treatment for a particular H II region (that is, a particular combination of density, luminosity, SFR, metallicity). However, each point could also be a galaxy, since a galaxy is an inhomogeneous container of possibly very different $\mathrm{H}$ II regions. Thus, every galaxy has its particular history, which may include accretion/stripping/collision episodes, so any point in the diagnostic diagrams reflects these. As we show in Fig. 6 the case (i) post-collisional models do not cross the data. However, reducing the temperature of the emitting source (in other words going to cases (ii) and (iii) in Figs. 6a,b) produces a shift toward the observations, and the models cross the data. In Fig. 6a, [O II] is more sensitive to the temperature than [O III] and increases with lower $T_{\text {eff. }}$ From Fig. 6b the ratio [O II] $(\lambda$ 3727)/H $\beta$ increases for lower temperatures. The shift of the model toward the data also occurs for the more dilute case (i) (i.e. $r_{\text {in }}=100 \mathrm{pc}$ ). For [O I] vs. [O III], we see most clearly the effect of our choice of stellar continuum. The higher ionization line ratios are quite sensitive to the history of both the luminosity (star formation rate) and metal abundances and, in general, the simple model works well. When a lower ion, or neutral atom, is used, the models systematically deviate, since the continuum fails to reproduce correctly the ionization stage of the gas. It is thus possible to separate these complementary effects and perhaps also see the indications of changes in the IMF with time. 

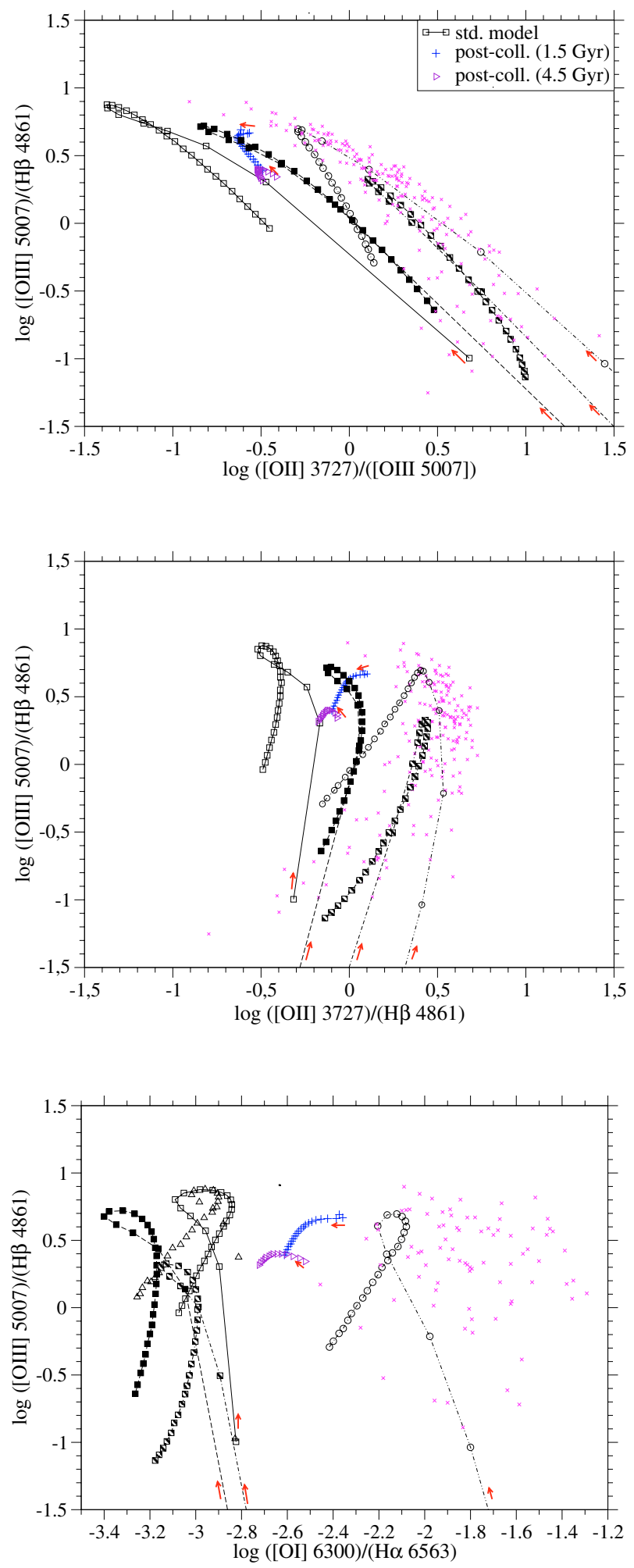

Fig. 6. Diagnostic diagrams: a) $\log ([\mathrm{O} \mathrm{III}] / \mathrm{H} \beta$ vs. $\log [\mathrm{O} \mathrm{II}] /[\mathrm{O} \mathrm{III}]$; b) $\log [\mathrm{O} \mathrm{III}] / \mathrm{H} \beta$ vs. $\log [\mathrm{O} \mathrm{II}] / \mathrm{H} \beta ;$ c) $\log [\mathrm{O} \mathrm{III}] / \mathrm{H} \beta$ vs. $\log ([\mathrm{O} \mathrm{I}] / \mathrm{H} \alpha$. Symbols: (blue, plus) post-collision, the collision started at $t_{\text {start }}=$ $1.5 \mathrm{Gyr}$; (violet, triangle right) post-collision, the collision started at $t_{\text {start }}=4.5 \mathrm{Gyr}$; (black, triangle up) metal-rich $\left(Z=Z_{\odot}\right)$ stochastic accretion by $25 \%$; all these models refer to the case (i) of Sect. 2. For the other symbols, see the correspondence symbols-models in the caption of Fig. 5. The red arrow near each model goes from earlier toward later times of the simulation. The magenta crosses are observations, from van Zee et al. (1998).

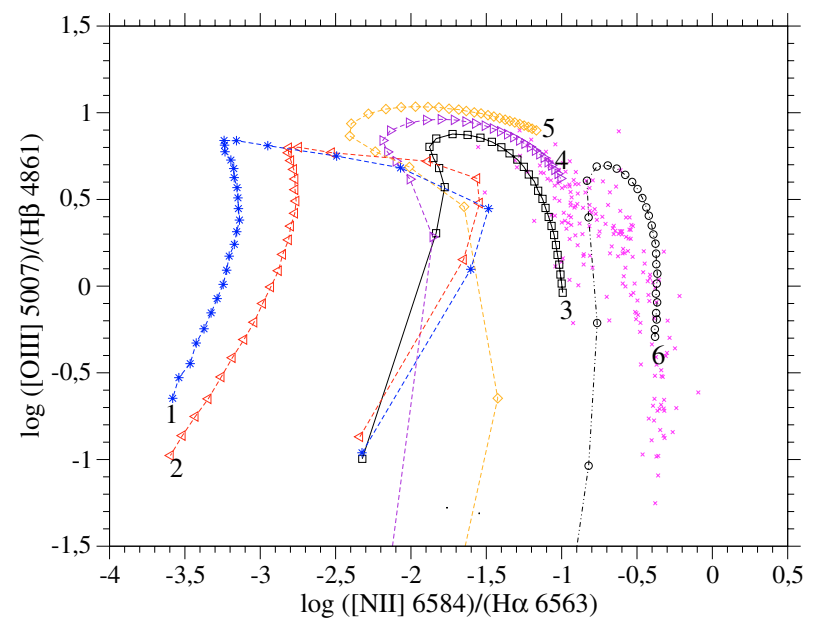

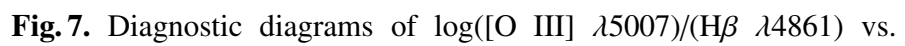
$\log ([\mathrm{N} \mathrm{II}] \lambda 6584) /(\mathrm{H} \alpha \lambda 6563)$, in the standard model, case (i), for different values of the density $n_{\mathrm{m}}$ in Eq. (4:) (1, blue stars) $5 \mathrm{~cm}^{-3} ;(2$, red, triangle left) $10 \mathrm{~cm}^{-3}$; (4, violet, triangles right) $1000 \mathrm{~cm}^{-3}$; (5, orange, diamonds) $10000 \mathrm{~cm}^{-3}$. For the density of $100 \mathrm{~cm}^{-3}$ : (3, squares) case (i); (6, circles) case (i), with $r_{\text {in }}=100 \mathrm{pc}$. The numbers at the end of each model, i.e. at the end of each time sequence, indicate the latest time.

To have an idea of the range spanned with the gas density variation, Fig. 7 shows the standard model for different densities as in Eq. (3), with $n_{\mathrm{m}}=$ from 5 to $10^{4} \mathrm{~cm}^{-3}$ in Eq. (4). For $100 \mathrm{~cm}^{-3}$ the case with larger inner radius $\left(r_{\text {in }}=100 \mathrm{pc}\right)$ is also displayed. The last time step of each sequence is indicated with a number in the plot. From this plot, we note that the time sequences of low density models lies near the data only at earlier times, while for later times they have lower values of $\log [\mathrm{N} \mathrm{II}] / \mathrm{H} \alpha$ and occupy an unexplored region of the diagram.

\subsection{Discussion}

Using a schematic representation of the stellar radiative input we have predicted the sensitivity of the emission line evolution to properties of the galactic models. Although it is well known and understood that the incident ionizing flux from imbedded hot stars is neither a Planck function nor independent of the stellar metal abundance, we find that the predominant species used for plasma diagnostics do not display a strong sensitivity to the details. To produce quantitative comparisons between the models and the observational data requires a more precise treatment of the line formation and galactic structure than we have used here and is not the point of this exploration; with only the thin disk explicitly in the photoionization modeling, we have not computed total emission line fluxes from all diffuse gas. It is possible, however, to extend this treatment to different radial and off-plane regions but this requires some caution ${ }^{2}$.

${ }^{2}$ That is, the disk is a relatively high density region and the recombination time is substantially lower than the evolutionary time. For the halo, because of its lower density and the long recombination time, a more precise treatment of the time dependent problem is required. For instance, for each model with Cloudy at any time $t$, we should use as input the ionization structure of the previous time step, $t-\Delta t$. 


\section{Conclusion}

In this series we have explored the phenomenological results of the CP approach to galactic star formation and chemical evolution modeling under a wide variety of physical conditions. In this paper, we have completed the study by interfacing the galactic code with a photoionization code to compare the results with more approximate treatments of star formation and chemical history. We have not tried to precisely model any particular system. Instead, our purpose has been to understand what aspects of the observations may reflect a generic feature of the nonlinear processes instead of detailed modeling of the photoionization inputs. The agreement we show with the observational data thus argues that a coupled treatment is essential for predictions of the emission line diagnostics. This suggests that, if the metallicity history is self-consistently derived from the galactic evolution model, many of the details of the spectral energy distribution may not be needed to get a picture of the history of the gas.

Acknowledgements. We thank Jose Franco, Joachim Köppen, Vladimir Korchagin, Jan Palouš, Greg Schwarz, and Lisa Van Zee for very helpful discussions and correspondence and our referee, Jesper Sommer-Larsen, for valuable comments and pointed questions. G.V. thanks Matteo Dell'Omodarme for precious advice, support, and encouragement. This work was supported, in part, by MIUR COFIN 2003.

\section{References}

Balick, B., \& Sneden, C. 1976, ApJ, 208, 336

Ferland, G. J. 1996, Hazy, A brief Introduction to Cloudy (University of Kentucky, Department of Physics and Astronomy, Internal Report)
Ferrini, F., Matteucci, F., Pardi, M. C., \& Penco, U. 1992, ApJ, 387, 138 Fioc, M., \& Rocca-Volmerange, B. 1999 [arXiv: astro-ph/9912179] Kennicutt, R. C. Jr. 1983, ApJ, 272, 54

Kennicutt, R. C. Jr. 1998, ARA\&A, 36, 189

Kewley, L. J., Dopita, M. A., Sutherland, R. S., Heisler, C. A., \& Trevena, J. 2001, ApJ, 556, 121

Kewley, L. J., Geller, M. J., \& Jansen, R. A. 2004, ApJ, 127, 2002

Köppen, J., \& Hensler, G. 2005, A\&A, 434, 531

Kobulnicky, H. A., Kennicutt, R. C., \& Pizagno, J. L. 1999, ApJ, 514, 544

Lehnert, M. D., \& Heckman, T. M. 1994, ApJ, 426, L27

Leitherer, C., et al. 1999, ApJS, 123, 3

Maier, C., Lilly, S. J., Carollo, C. M., et al. 2006, ApJ, 639, 858

Martins, L. P., et al. 2005, MNRAS, 358, 49

Moustakas, J., Kennicutt, R. C. Jr., \& Tremonti, C. A. [arXiv: astro-ph/0511730]

Moy, E., Rocca-Volmerange, B., \& Fioc, M. 2001, A\&A, 365, 347

Osterbrock, D. E., \& Ferland, G. J. 2006, Astrophysics of Gaseous Nebulae and Active Galactic Nuclei, Second Edition (Sausalito, California: University Science Books)

Quilis, V., Moore, B., \& Bower, R. 2000, Science, 288, 1617

Rocha-Pinto, H. J., Flynn, C., Scalo, J., et al. 2004, A\&A, 423, 517

Shore, S. N. 2003, The Tapestry of Modern Astrophysics (Hoboken, NJ: Wiley-Interscience)

Shore, S. N., \& Ferrini, F. 1995, Fund. Cosm. Phys., 16, 1

Sutherland, R. S., \& Dopita, M. A. 1993, ApJS, 88, 253

Stasinska, G., \& Izotov, Y. 2003, A\&A, 397, 71

Vacca, W. D., Garmany, C. D., \& Shull, J. M. 1996, ApJ, 460, 914

Valle, G., Ferrini, F., Galli, D., \& Shore, S. N. 2002, ApJ, 566, 252 (VFGS02)

Valle, G., Shore, S. N., \& Galli, D. 2005a, A\&A, 435, 551 (VSG05)

Valle, G., Cignoni, M., \& Shore, S. N. 2005b, A\&A, 440, 473 (VCS05)

van Zee, L., Salzer, J. J., Haynes, M. P., O’Donoghue, A. A., \& Balonek, T. J. 1998, AJ, 116, 2805

van Zee, L., \& Haynes, M. P. 2006, ApJ, 636, 214

Zackrisson, E., Bergvall, N., Olofsson, K., \& Siebert, A. 2001, A\&A, 375, 814 